\title{
Influência do local da anastomose (cervical ou torácica) na morbi-mortalidade das esofagectomias
}

\author{
Influence of site of anastomosis (cervical or thoracic) on morbidity and mortality \\ from esophagectomies
}

\author{
Flávio Daniel Saavedra Tomasich, ${ }^{1}$ Gerardo Cristino Gavarrette Valladares, ${ }^{2}$ \\ Viviane Coimbra Augusto Demarchi e Danilo Gagliardi ${ }^{4}$
}

\begin{abstract}
Resumo
O bjetivo: avaliar a influência do local da anastomose (cervical ou torácica) nas complicações pós-operatórias e mortalidade das esofagectomias com linfadenectomia em dois campos. Métodos: Estudo retrospectivo de 132 pacientes submetidos a esofagectomia com anastomose cervical ou intratorácica no $D$ epartamento de Cirurgia do H ospital Erasto Gaertner de janeiro/1987 a janeiro/1998. Analisaram-se variáveis relativas ao paciente (sexo, idade, estado geral, perda ponderal, co-morbidades, tabagismo, risco pulmonar), ao tumor (tipo histológico, localização, estádio clínico) e ao procedimento cirúrgico (tipo da anastomose, tempo cirúrgico, tempo de hospitalização), relacionando-as com as complicações e mortalidade pós-operatórias. Resultados: N oventa e quatro pacientes $(71,2 \%)$ eram do sexo masculino. 0 tipo histológico predominante foi o carcinoma espino-celular (CEC) em $94,7 \%$ dos casos. As principais co-morbidades anotadas foram doença pulmonar obstrutiva crônica (D POC) $(29,55 \%)$ e hipertensão arterial sistêmica (H AS) $(15,15 \%)$, e 88 pacientes $(66,6 \%)$ eram tabagistas. A principal localização do tumor foi o segmento torácico inferior $(56,06 \%)$. Seis pacientes $(4,54 \%)$ eram de estágio clínico (EC) I, 44 (33,33\%) IIA, 24 (18,18\%) IIB, 38 (28,80\%) III e 17 (12,90\%) IV. A anastomose intratorácica foi realizada em 105 pacientes (79,55\%) e cervical em 27 (20,45\%). A taxa de complicações foi de 39,3\% e a letalidade hospitalar 13,70\%. Procedeu-se anastomose mecânica em $65,09 \%$ dos casos e manual em 39,91\%. 0 correram seis casos $(23,1 \%)$ de fístula cervical e três $(2,9 \%)$ de intratorácica $(p=0,002)$. A mortalidade específica foi de 33,3\% nos dois subgrupos. Conclusão: Este estudo mostrou uma maior ocorrência de fístulas nas anastomoses cervicais. A mortalidade pós-operatória foi semelhante nas duas técnicas, contrariando a tendência da literatura de conferir às fístulas cervicais uma menor letalidade.

Palavras-chave: neoplasias esofágicas; esofagectomia; anastomose cirúrgica; complicações pós-operatórias; mortalidade; fístula.
\end{abstract}

\footnotetext{
${ }^{1}$ Cirurgião O ncológico do Serviço de Cirurgia Abdominal do Hospital Erasto Gaertner. Enviar correspondência para F.D.S.T. D epartamento de Cirurgia do H ospital Erasto Gaertner (HEG) da Liga Paranaense de Combate ao Câncer, Rua Dr O vande do Amaral 201, Jardim das Américas; 81520-060 Curitiba, PA - Brasil.

${ }^{2}$ Chefe do Serviço de Tórax do H ospital Erasto Gaertner, Curitiba, PA - Brasil.

${ }^{3}$ Cirurgiã O ncológica do H ospital Erasto Gaertner, Curitiba, PA - Brasil.

${ }^{4}$ Chefe do Serviço de Esôfago do D epartamento de Cirurgia da Faculdade de Ciências M édicas da Santa Casa de São Paulo, São Paulo, SP - Brasil.
} 


\begin{abstract}
Background. To evaluate the influence of the site of the anastomosis (cervical or intrathoracic) on postoperative complications and mortality from esophagectomies with two-field lymphadenectomy. M ethods. Retrospective study of 132 patients with esophageal cancer submitted to esophagectomy with cervical or intrathoracic anastomosis admitted in D epartment of Surgery in Erasto Gaertner H ospital from January 1987 to January 1998. Patientrelated variables (gender, age, overall condition, weight loss, co-morbidities, tabagism), tumor-related variables (histological type, site, staging), and to surgical procedure variables (type and site of anastomosis, surgical time, hospitalization time) were recorded and related to postoperative complications and mortality. Results. N inety-nine patients $(71.2 \%)$ were males. The predominant histological type was squamous cell carcinoma, in $94.7 \%$ of the cases. The main co-morbidities reported were chronic obstructive pulmonary disease $(29.55 \%)$ and arterial hypertension $(15.15 \%)$, and 88 patients $(66.6 \%)$ were smokers. The main tumor site was the inferior thoracic segment $(56,06 \%)$. Six patients (4.54\%) were stage I, $44(33.33 \%)$ IIA, $24(18.18 \%)$ IIB, $38(28.80 \%)$ III and $17(12.90 \%)$ IV. The intrathoracic anastomosis was performed in 105 patients $(79.55 \%)$, and cervical in $27(20.45 \%)$. The complication rate was $39.3 \%$ and hospital mortality, $13.70 \%$. M echanical anastomosis was performed in $65.09 \%$ of cases, and manual in $39.91 \%$. Cervical leak occurred in 6 patients $(23.1 \%)$, and $3(2.9 \%)$ had intrathoracic leak $(p=0.002)$. The specific mortality was $33.3 \%$ in both subgroups. Conclusion. This study showed a higher occurrence of leakage in cervical anastomosis. The postoperative mortality was equal in both techniques, contradicting the literature tendency to attribute lower lethality to cervical leakage.
\end{abstract}

Key words esophageal neoplasms; esophagectomy; surgical anastomosis; postoperative complications; mortality; leakage.

\section{INTRO DUÇÃO}

Desde a década de 40, quando 0 shawa e Adams iniciaram a era da reconstrução imediata na cirurgia do esôfago após a ressecção do segmento torácico, já surgiram diferentes correntes defendendo a realização da anastomose na região cervical ou intratorácica. ${ }^{1} \mathrm{~A}$ manobra de Kocher, com secção dos vasos curtos e tronco da artéria gástrica esquerda, facilita a mobilização do estômago e duodeno e possibilita as anastomoses altas com redução da tensão ao nível da anastomose cervical. $^{2}$

Em 1946, Ivor Lewis popularizou a técnica que consiste em combinar uma toracotomia direita e laparotomia para a ressecção do câncer de esôfago, seguida de esofagogastrostomia intratorácica. ${ }^{1}$

As deiscências das anastomoses são referidas como complicações freqüentes do câncer do esôfago operado. As cervicais são consideradas mais freqüentes que as torácicas porém de menor gravidade e evolução mais benigna. ${ }^{3-7}$

Em relação às complicações pós-operatórias, independentemente do tipo de cirurgia realizada, as de natureza respiratória e as deiscências de anastomose são as mais freqüentemente referidas na literatura. ${ }^{3-5,7-9}$

Entre os fatores de risco que podem influenciar a ocorrência das complicações pós-operatórias e letalidade hospitalar da cirurgia do câncer do esôfago, são citados: idade, sexo, grau de disfagia, doenças associadas, localização e extensão do tumor, tipo histológico, estado nutricional e deficiência imunológica, quimioterapia e radioterapia, cirurgia realizada, tipo de anastomose (manual ou mecânica), local da anastomose (cervical ou torácica), perda sangüínea operatória, caráter da cirurgia, estádio da doença, complicações pleuro-pulmonares, deiscências das anastomoses e reoperações. ${ }^{9-11}$ Tentando correlacionar alguns dos fatores relatados com as complicações pós-operatórias imediatas e a letalidade hospitalar, obtiveram resultados diversos, em virtude da seleção de diferentes variáveis e casuísticas não comparáveis.

Assim sendo, reunimos 132 pacientes portadores de câncer do esôfago submetidos à esofagogastrectomia com o objetivo de comparar as complicações pós-operatórias e a letalidade hospitalar em relação ao tipo de anastomose realizada (cervical ou torácica), analisando sexo, idade, estado geral, localização do tumor, grau de comprometimento pulmonar, tipo da anastomose e estádio patológico.

\section{MATERIAL E MÉTO DOS}

Foram avaliados 132 pacientes portadores de câncer do esôfago admitidos no D epartamento de Cirurgia do H ospital Erasto $\mathrm{G}$ aertner no período de janeiro de 1987 até janeiro de 1998 . Todos submetidos à rotina de exames pré-operatórios e estadiados de acordo com as normas de classificação TN M da União Internacional 
de Combate ao Câncer (UICC). Foram incluídos no estudo pacientes com câncer de esôfago com confirmação histológica, localizados nos segmentos abdominal e torácico superior, médio ou inferior, com até $7 \mathrm{~cm}$ de extensão, sem desvio significativo do eixo, sinus e trajeto fistuloso ao esofagograma e esofagoscopia, sem sinais de invasão por contigüidade ou disseminação a distância. Foram excluídos os tumores da região cervical.

O s pacientes selecionados foram submetidos à esofagectomia, com ressecção macroscópica completa do tumor em todos casos, e linfadenectomia abdominal e mediastinal concomitante (cadeias paraesofágica, paratraqueal, subcarinal, paracardiais, tronco celíaco, pequena e grande curvatura do estômago), através de laparotomia mediana xifoumbilical, toracotomia direita no quinto espaço intercostal e cervicotomia lateral esquerda nas anastomoses cervicais. Efetuamos quatro tipos de reconstrução do trânsito: esôfago-gástrica intratorácica, esôfago-gástrica cervical, esôfago-cólica cervical e esôfago-jejunal intratorácica, com anastomose manual ou mecânica. Foram submetidos a tratamento neoadjuvante os pacientes com tumores T 3 e com suspeita de metástases linfonodais regionais aos exames de imagem, totalizando 61 casos.

Foram anotadas as seguintes variáveis: sexo, idade, estado geral, através das escalas de Zubrod e Karnofsky Performance status, grau de desnutrição, perda ponderal, co-morbidades, tabagismo, risco pulmonar, tipo histológico, localização do tumor, estádio clínico, tipo da anastomose, tempo cirúrgico, tempo de permanência hospitalar, complicações pós-operatórias e letalidade hospitalar, sendo relacionadas com a realização cervical ou intratorácica da anastomose.

Foram consideradas como complicações pósoperatórias quaisquer intercorrências, isoladas ou múltiplas, de natureza diversa, que necessitaram tratamento clínico, cirúrgico ou associado dependendo de sua gênese, ocorridas no período de 30 dias após o ato cirúrgico. Foram agrupadas em pleuro-pulmonares, cardiovasculares, fístulas, infecciosas, neurológicas, hemorrágicas, quilotórax, insuficiência renal. A mortalidade pós-operatória foi definida como os óbitos ocorridos até 30 dias após 0 ato cirúrgico ou após 30 dias quando decorrentes de complicações pósoperatórias.

O s resultados foram submetidos à análise estatística descritiva das variáveis. Para comparação entre percentuais foi utilizado o teste de diferenças entre percentuais. Procedeu-se a análise univariada em que cada uma das variáveis explanatórias foi correlacionada individualmente com as variáveis resultantes, utilizandose para tal finalidade 0 "Coeficiente de Contingência
C". Realizada a analise multivariada, pela Regressão Logística M últipla, com o objetivo de verificar as variáveis explanatórias analisadas em conjunto, em relação a cada uma das variáveis resultantes. 0 nível de significância adotado foi de $5 \%(p \varangle 0,05)$ para todos os testes.

\section{RESULTADO $S$}

N oventa e quatro pacientes $(71,2 \%)$ eram do sexo masculino e $38(28,8 \%)$ do feminino. Q uanto ao tipo histológico verificamos: 125 (94,7\%) casos de carcinoma de células escamosas, seis casos de adenocarcinoma $(4,5 \%)$ e um caso de carcinossarcoma $(0,8 \%)$.

Em relação à escala de Karnofsky, 95 (72\%) dos pacientes apresentava escore igual ou superior a $80 \%$, $36(27 \%)$ índiceigual a $70 \%$ eapenasum $(0,75 \%)$ menor que $70 \%$. N a escala de Zubrod, quatro pacientes (3\%) apresentavam escore 0; $86(65,1 \%), 1 ; 36(27,2 \%)$, dois e seis $(4,5 \%), 3$. O itenta e três pacientes $(63 \%)$ apresentavam algum grau de desnutrição. Emagrecimento esteve presente em $94,7 \%$ da amostra, com perda ponderal de $10 \%$ em 37 pacientes $(29,6 \%)$, de $10 \%$ a $20 \%$ em $43(34,4 \%)$ e acima de $20 \%$ em 45 (36\%).

Trinta e nove pacientes $(29,55 \%)$ tinham doença pulmonar obstrutiva crônica (DPOC) relatada e confirmada pelos exames complementares; $15,15 \%$ tinham hipertensão arterial sistêmica (H AS); 6,06\%, hiper-sensibilidade a algum tipo de medicamento e $1,51 \%$, megaesôfago. 0 itenta e oito pacientes $(66,67 \%)$ eram tabagistas. Setenta e três por cento dos pacientes apresentava risco pulmonar I e II, e $27 \%$ risco III e IV, considerados como risco moderado e grave.

A localização dos tumores foi em segmento torácico inferior em 74 pacientes $(56,06 \%)$, torácico médio em $55(41,67 \%)$ e torácico superior em $3(2,27 \%)$, com média da extensão tumoral de 47,72 mm, variando de 8 a $70 \mathrm{~mm}$. Em relação ao estadiamento do tumor, 11 pacientes $(8,33 \%)$ eram T 1, $43(32,58 \%)$, T $2 ; 58$ $(43,94 \%)$, T 3 e $12(9,09 \%)$ T 4 . O s linfonodos encontraram-se comprometidos em 72 casos $(54,54 \%)$ e havia metástases em 17 (12,90\%). Assim sendo, seis pacientes (4,54\%) eram EC I, 44 (33,33\%) EC IIA, 24 $(18,18 \%)$ EC IIB, $38(28,80 \%)$ EC III e 17 (12,90\%) EC IV. Três pacientes não foram estadiáveis.

Procedeu-se anastomose mecânica em 65,09\% dos casos e manual em $39,91 \%$. 0 tempo cirúrgico médio foi de 342,7 minutos, oscilando entre 180 até 600 minutos. A média global de permanência hospitalar foi de 20,5 dias e a pós-operatória de 15,8 dias.

A anastomose esôfago-gástrica intratorácica foi 
realizada em 103 pacientes (78,04\%), seguida pela esôfago-gástrica cervical em 26 (19,70\%), esôfago-jejunal intra-torácica em dois (1,5\%) e coloplastia em um $(0,75 \%)$. (Tabela 1$)$
Complicações pós-operatórias, isoladas ou múltiplas, ocorreram em 52 pacientes (39,3\%), sendo especificadas naTabela 2. A letalidade hospitalar global foi de 13,70\%, sendo especificadas as causas de óbitos na Tabela 3.

Tabela 1. Distribuição dos pacientes segundo o local da anastomose.

\begin{tabular}{|c|l|c|}
\hline $\begin{array}{c}\text { Localização da } \\
\text { anastomose }\end{array}$ & \multicolumn{1}{|c|}{ Tipo de anastomose } & N (\%) \\
\hline Cervical & $\begin{array}{l}\text { Anastomose esôfago-gástrico cervical }(n=26) \\
\text { Coloplastia }(n=1)\end{array}$ & $27(20,45 \%)$ \\
\hline Torácica & $\begin{array}{l}\text { Anastomose esôfago-gástrica }(n=103) \\
\text { Anastomose esôfago-jejunal }(n=2)\end{array}$ & $105(79.55 \%)$ \\
\hline
\end{tabular}

Tabela 2. Tipos de complicações pós-operatórias $(n=52)$.

\begin{tabular}{|l|c|}
\hline \multicolumn{1}{|c|}{ Complicação } & N (\%) \\
\hline Pleuro-pulmonar & $25(48,08 \%)$ \\
\hline Cardiovascular & $9(17,31 \%)$ \\
\hline Fístula anastomótica & $9(17,31 \%)$ \\
\hline Sépsis & $7(13,46 \%)$ \\
\hline Infecção de sítio cirúrgico & $6(11,54 \%)$ \\
\hline Abscesso intra-abdominal & $4(7,69 \%)$ \\
\hline Neurológica & $2(3,85 \%)$ \\
\hline Hemorragia & $2(3,85 \%)$ \\
\hline Q uilotórax & $2(3,85 \%)$ \\
\hline Insuficiência renal & $1(1,92 \%)$ \\
\hline
\end{tabular}

Tabela 3. Causas de óbito pós-operatório $(n=18)$.

\begin{tabular}{|l|c|}
\hline \multicolumn{1}{|c|}{ Causa do óbito } & N (\%) \\
\hline Pleuro-pulmonar & $8(44,44 \%)$ \\
\hline Cardiovascular & $5(27,78 \%)$ \\
\hline Fístula anastomótica & $3(16,67 \%)$ \\
\hline Sépsis & $2(11,11 \%)$ \\
\hline
\end{tabular}

Q uando o local da anastomose era a região cervical, ocorreram seis $(23,1 \%)$ casos de fístula comparativamente a três $(2,9 \%)$ da anastomose intratorácica, sendo essa diferença altamente significativa, com $p=0,002$ (Figura 1). A letalidade hospitalar destes pacientes com fístulas foi de dois óbitos em pacientes com anastomose cervical e um com anastomose intratorácica.

A confecção da anastomose foi mecânica em 87 pacientes $(65,09 \%)$ e manual em $45(34,91 \%)$. Em relação às fístulas, estas ocorreram em $13,3 \%$ quando a anastomose foi confeccionada manualmente, comparativamente a $3,44 \%$ nas realizadas com sutura mecânica, não sendo estatisticamente significante $(p=0,12)$. $N$ as anastomoses manuais cervicais ocorreram fístulas em $21,7 \%$, contra $4,55 \%$ nas manuais intratorácicas, não sendo estatisticamente significante 
( $p=0,0971) . N$ as confeccionadas mecanicamenteao nível cervical, a proporção de fístulas foi $25 \%$ contra $2,41 \%$ nas mecânicas intratorácicas, não sendo estatisticamente significante. (Figura 2)

$\mathrm{Na}$ análise univariada e multivariada, a correlação das variáveis (sexo, idade, Zubrod, Karnofsky Peformance satus, localização do tumor, risco pulmonar, tipo da anastomose, estadio clínico) com o local da anastomose (cervical ou torácica) não foram consideradas estatisticamente significantes.

Figura 1. Distribuição das fístulas em relação ao local da anastomose

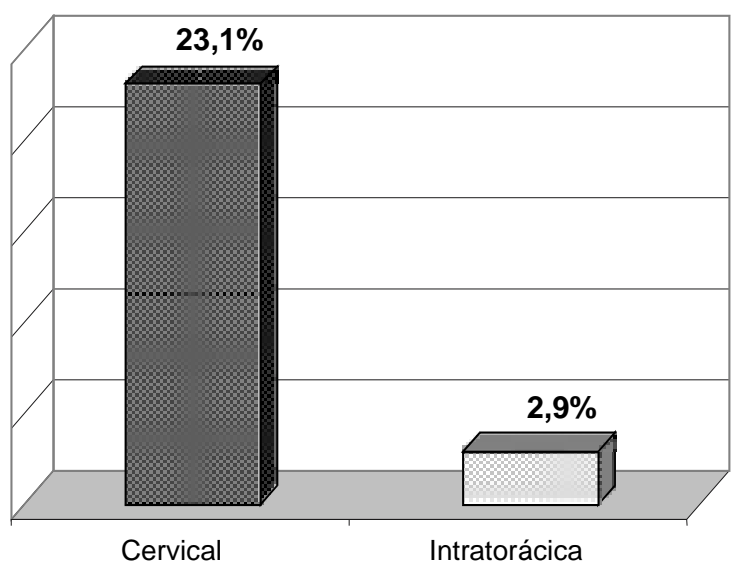

Figura 2. Distribuição das fístulas em relação ao local e tipo de anastomose.

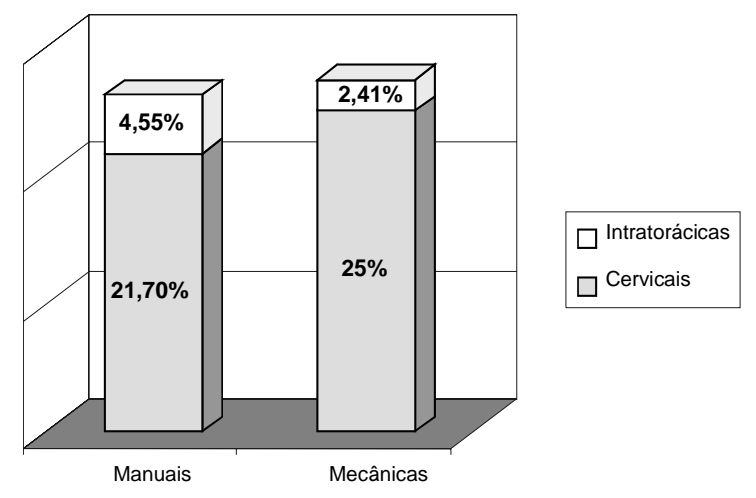

\section{DISCUSSÃO}

As abordagens mais utilizadas para a realização das esofagectomias podem ser parcialmente categorizadas pela localização da anastomose esôfago-gástrica: torácica ou cervical. A confecção de uma anastomose cervical pode ser realizada por acesso trans-hiatal ou combinado, através de acessos abdominal, torácico e cervical. ${ }^{12} \mathrm{~A}$ esofagectomia trans-hiatal foi difundida por 0 rringer $\mathrm{e}$ Sloan ${ }^{13}$ e tem como vantagem evitar a toracotomia, combinando uma incisão cervical com uma laparotomia, devendo ser evitada nos casos de suspeita de invasão da aorta ou da bifurcação traqueobrônquica. A crítica em relação a essa abordagem é a limitação em relação à dissecção linfonodal. ${ }^{12}$ Se for preferida a visualização direta do esôfago torácico, a dissecção no tórax pode ser realizada conforme a técnica de $M$ cK eown, ${ }^{8}$ que combina a laparotomia com toracotomia e cervicotomia direitas. A anastomose cervical tem a vantagem de permitir uma dissecção linfonodal extendida e as eventuais complicações geralmente são mais bem toleradas e com menor morbidade que as anastomoses torácicas. Entretanto, a extensão do conduto para substituição do esôfago até a região cervical pode aumentar o risco de fístulas anastomóticas, estenose, isquemia do conduto e lesão do nervo laríngeo recorrente. ${ }^{6,7}$

A anastomose intratorácica pode ser confeccionada por acesso transtorácico esquerdo ou por uma laparotomia associada à toracotomia direita, conhecida como esofagogastrectomia à Ivor-Lewis. Essa técnica permite uma ressecção esofágica parcial ou subtotal e fornece exposição para uma completa dissecção linfonodal regional, estando indicada para tumores dos terços médio e inferior do esôfago e cárdia. ${ }^{1,12}$ Classicamente, a ocorrência de fístulas anastomóticas nessa técnica é menor que nas anastomoses cervicais. É provável que a cirurgia realizada com anastomose intratorácica, sem nenhum grau de tensão, seja responsável pelo menor número de fístulas. 4,9,14

0 maior argumento em favor da anastomose cervical é a potencial menor morbi-mortalidade de uma fístula nessa localização. Embora a maioria dos autores aceite que as anastomoses cervicais resultam em maiores taxas de deiscência, ${ }^{3,4,6}$ afirmação contestada por outros autores, ${ }^{15}$ as conseqüências dessas fístulas tendem a ser menos danosas que uma fístula intratorácica. ${ }^{7}$ Embora isso seja verdade em um grau, uma avaliação cuidadosa da literatura recente revela um dramático decréscimo na mortalidade global associada às fístulas intratorácicas e complicações catastróficas relacionadas às anastomoses cervicais. Talvez o reconhecimento precoce das complicações e uma intervenção cirúrgica agressiva, tenham contribuído para essa redução substancial da mortalidade. $^{12}$

A conduta de escolha dos autores para o tratamento dos tumores de terço médio e inferior na Instituição é a esofagectomia com toracotomia direita, realizada em todos os pacientes incluídos neste estudo. Esta via de acesso permite uma melhor abordagem do tumor e dos linfonodos regionais com menor risco de lesão de estruturas mediastinais, uma anastomose mais segura, 
livre de tensão, e menos lesão tecidual no conduto pelo menor manuseio, com uma morbi-mortalidade aceitável e decrescente com a experiência.

$\mathrm{N}$ a experiência de Agrawal et al, ${ }^{14}$ baseados na revisão de 111 pacientes submetidos a esofagectomia à Ivor-Lewis, o acesso transtorácico com anastomose intratorácica é um procedimento com baixa mortalidade e morbidade aceitável. A mortalidade global foi de apenas $1,8 \%$ e a incidência de fístula anastomótica, $9 \%$ com taxa de mortalidade dessas fístulas em $20 \%$. Corroborando esses dados, 0 estudo de Sauvanet et al ${ }^{16}$ com 409 pacientes submetidos à esofagectomia (358 Ivor-Lewis e 51 transtorácica esquerda) mostra uma mortalidade global de 2,2\% e uma incidência de fístula de $9,2 \%$ (38 pacientes), com nove óbitos nesse subgrupo, representando uma mortalidade específica de $23 \%$ para os casos de fístulas intratorácicas. $N$ akadi et al ${ }^{9}$ relataram 119 pacientes operados, sendo $98 \%$ dos casos com anastomose intratorácica. A letalidade hospitalar registrada foi de $5,9 \%$ e $35 \%$ de complicações, sendo que as fístulas anastomóticas aconteceram em $2 \%$ dos casos.

Em outra série de $\mathrm{Karl}$ et $\mathrm{al}^{17}$ que incluiu 143 pacientes submetidos a esofagectomia à Ivor-Lewis, os autores apresentam uma taxa de fístula de 3,5\%, porém três dos cinco pacientes que apresentaram essa complicação evoluíram a óbito, representando uma mortalidade superior a outros estudos (60\%), apesar da baixa incidência de fístula. 0 estudo de Whooley et al ${ }^{18}$ aponta a mesma incidência de fístulas e uma mortali idade de 8,9\%, numa casuística de 710 esofagectomias, sendo $83 \%$ por abordagem transtorácica. Embora este último estudo inclua também pacientes operados por outras abordagens, a análise de regressão logística não apontou essa variável como significativa na ocorrência de óbitos pós-operatórios. Essas séries ainda indicam a gravidade das fístulas intratorácicas, mas mostram uma redução da mortalidade decorrente dessa complicação com 0 passar dos anos, o que provavelmente reflete um diagnóstico adequado e tratamento apropriado.12

A incidência de fístulas nas anastomoses cervicais na literatura varia de $10 \%$ a $23 \%$, com uma mortalidade global entre $2 \%$ e $9 \%$. 5,7,19,20 O utras complicações freqüentes nas anastomoses cervicais são as estenoses recorrentes e lesão do nervo laríngeo recorrente. A taxa de estenose varia de $5 \%$ a $50 \%$, sendo significativamente aumentada quando a anastomose é localizada na posição cervical. 7,12,20,21 I sso pode ser fisiologicamente relacionado à isquemia decorrente do posicionamento de um tubo gástrico extenso até o pescoço, com conseqüente tensão na anastomose, podendo resultar inclusive em gangrena do conduto. ${ }^{12}$ Essa complicação catastrófica tem uma incidência que varia de menos de $1 \%$ a 3,9\%, porém com uma letalidade que pode chegar a $97 \%, 7,22$

N este estudo, obtivemos um maior índice de fístulas nas anastomoses cervicais $(23,1 \%)$ quando comparadas às fístulas intratorácicas (2,9\%). Astaxas são semelhantes às descritas pelos diferentes autores. A letalidade hospitalar foi elevada tanto nas fístulas cervicais como nas intratorácicas, 33,3\% em ambas. Consideramos a localização da anastomose um tópico significativo e pela alta letalidade das fístulas cervicais nesta casuística, questionamos a consideração de que estas sejam inócuas.

O utra grave complicação dessa técnica é 0 sangramento mediastinal, intra ou pós-operatório. $\mathrm{Na}$ série de 0 rringer et al ${ }^{7}$ com 1085 pacientes, ocorreram seis casos de sangramento mediastinal intra-operatório, com mortalidade intra-operatória de 50\%. Embora não tenha sido descrito como foi realizado o controle dessa complicação, esses pacientes não foram submetidos à toracotomia, uma vez que foram excluídos do estudo 15 pacientes que necessitaram conversão por fixação ou sangramento mediastinal. $0 \mathrm{~s}$ autores apenas descrevem que com a experiência passaram a realizar a mobilização do esôfago sob visualização direta ao invés de dissecção romba. Outros cinco pacientes apresentaram sangramento num período de 24 horas de pós-operatório e necessitaram toracotomia para controle do sangramento. Em nossa concepção, além da dissecção romba aumentar o risco de sangramento mediastinal, esse tipo de abordagem resulta num tratamento inadequado dos linfonodos regionais.

Em termos de sobrevida e princípios oncológicos, 0 papel da anastomose intratorácica deve ser analisado em relação à extensão da margem de ressecção esofágica e da linfadenectomia. A margem de ressecção no câncer de esôfago mostra-se como um importante fator prognóstico de recorrência local. Segundo Tam et al, ${ }^{23}$ margens de ressecção menores que $10 \mathrm{~cm}$ estão associadas com maiores taxas de recorrência anastomótica, respectivamente: $25 \%, 15 \%$ e $7 \%$ para margens de $2 \mathrm{~cm}, 5 \mathrm{~cm}$ e $10 \mathrm{~cm}$.

$\mathrm{N} 0$ estudo de $\mathrm{K}$ ato et $a l,{ }^{24} \mathrm{com} 463$ pacientes submetidos à esofagectomia I vor-Lewis, demonstrouse uma crescente taxa de recorrência local relacionada com a localização do tumor primário no tórax, com recorrência anastomótica de 6\%, 3\% e 1\% para tumores localizados no terço superior, médio e inferior do esôfago, respectivamente. A maioria dos autores recomenda uma margem de $10 \mathrm{~cm}$, independente da localização da anastomose. Lesões esofágicas inferiores teoricamente deveriam ser ressecadas com anastomose 
torácica alta ou cervical, enquanto lesões localizadas no esôfago médio e superior requereriam, por necessidade de margem, uma anastomose cervical. Entretanto, não se justifica a realização de uma esofagectomia total para uma lesão de terço inferior do esôfago com anastomose cervical, fato comprovado por vários autores que não mostram nenhuma vantagem de sobrevida sobre a esofagectomia subtotal com anastomose intratorácica. ${ }^{25-27}$

Apesar das diferenças técnicas e oncológicas entre a esofagectomia trans-hiatal e transtorácica, vários estudos não mostram diferença na sobrevida a longo prazo. 6,22,26,27 As características do paciente e do tumor podem ajudar a definir a extensão e abordagem da ressecção. A decisão do cirurgião em usar uma ou outra técnica é influenciada pela localização do tumor, tamanho do tumor, possível envolvimento de estruturas adjacentes, necessidade de linfadenectomia, estado geral, irradiação prévia do esôfago, biótipo, além das preferências individuais do cirurgião.

\section{CO NCLUSÃO}

Esteestudo mostrou uma mai or ocorrência de fístulas nas anastomoses cervicais. A mortalidade pós-operatória especificamente relacionada à fístula foi semelhante nas duas técnicas, contrariando a tendência da literatura de conferir às fístulas cervicais uma menor letalidade.

Acreditamos que os resultados refletem a experiência de um centro especializado, resultando numa melhor seleção de pacientes para a abordagem torácica, contribuindo para a redução das complicações em pacientes submetidos à esofagectomia.

\section{REFERÊN CIAS BIBLIO G RÁFICAS}

1. LeeRB, M iller JO . Esophagectomy for cancer. Surg Clin N Am 1997;77(5):1169-96.

2. Sweet RH . Late results of surgical treatment of carcinoma of the esophagus. JAM A 1945;155:422-5.

3. Gagliardi D. C âncer do esôfago, complicações pósoperatóriasimediataseletalidadehospitalar [tese]. São Paulo (SP): Faculdade deC iências M édicas da Santa Casa deSão Paulo; 1994.

4. EllisFH Jr. Standard resection for cancer of theesophagus and cardia. Surg O ncol Clin N Am 1997;8(2):279-94.

5. M irraAP, Justo FA, Schneider CAR, TrippeN . Tratamento do câncer de esôfago: ensaio clínico controlado. Rev Col Bras Cir 1997;25(2):119-22.

6. Rindani R, M artin CJ, C ox M R. Transhiatal versus Ivor Lewis oesophagectomy: is there a difference? Aust N Z J Surg 1999;69(3):187-94.

7. O rringer $M B, M$ arshall $B$, Iannettoni $M D$. Transhiatal esophagectomy: clinical experienceand refinements. Ann Surg 1999;230(3):392-403.

8. M cKeown KC. Resection of midesophageal carcinoma with esophagogastric anastomosis. WorldJ Surg 1981;5(4):517-25.

9. N akadi I, H ouben JJ, G ay F, C losset J, G elin M , Lambilliotte JP. D oes esophagectomy cure a resectable esophageal cancer? World J Surg 1993;17(6):760-4.

10. Law S, Fok KM , C hu JW. Thoracoscopic esophagectomy for esophageal cancer. Surgery 1997;122(1):8-14.

11. BartelsH , Stein HJ, SiewertJ R. Risk analysisin esophageal surgery. Recent Results C ancer Res 2000;155:89-96.

12. Sonett JR. Esophagectomy. Therole of the intrathoracic anastomosis. Chest Surg Clin N Am 2000;10(3):519-30.

13. $O$ rringer $M B$, Sloan $H$. E sophagectomy without thoracotomy. J Thorac C ardiovasc Surg 1978;76(5):643-54.

14. Agrawal S, D eshmukh SP, Patil PK, Bhansali M S, Bhatt RG, BadweRA, et al. Intrathoracic anastomosis after oesophageal resection for cancer. J Surg O ncol 1996;63(1):52-6.

15. M CM anus K, Anikin V, M cGuigan J. Total thoracic oesophagectomy for oesophageal carcinoma: has it been worth it? Eur J Cardiothorac Surg 1999;16(3):261-5.

16. Sauvanet A, Baltar J, M ee JL. D iagnosis and conservative management of intrathoracic leakageafter oesophagectomy. Br J Surg 1998;85:1446-9.

17. Karl RC, Schreiber R, BoulwareD, Baker S, Coppola D . Factors affecting morbidity, mortality and survival in patients undergoing I vor Lewis esophagectomy. Ann Surg 2000;231:635-43.

18. Whooley BP, Law S, M urthy SC, Alexandou A, Wong J. Analysis of reduced death and complication rates after esophageal resection. Ann Surg 2001;233:338-44.

19. G andhi SK, N aunheim KS. Complications of transhiatal esophagectomy. Chest Surg Clin N Am 1997;7(3):601-10.

20. KatariyaK K, H arveyJC, PinaE, BeattieE. Complications of transhiatal esophagectomy. J Surg O ncol 1994;57:157-63.

21. H onkoop P, Siersema PD , Tilanus H W, Stassen LP, H op W C, van Blankenstein M . Benign anastomosisstrictures alter transhiatal esophagectomy and cervical esophagogastrostomy: risk factorsand management. J Thorac $C$ ardiovasc Surg 1996;111(6):1141-6.

22. M oorehead RJ, WongJ. G angrenein esophageal substitutes after resection and bypass procedures for carcinoma of the esophagus. H epatogastroenterology 1990;37(4):364-7.

23. Tam PC, Siu KF, Cheung H C, M aL, Wong J. Local recurrences after subtotal esophagectomy for squamouscell carcinoma. Ann Surg 1987;205:189-94.

24. $\mathrm{Kato} \mathrm{H}$, Tachimori Y, WatanabeH , Yamaguchi H , Ishikawa $\mathrm{T}, \mathrm{N}$ akanishi $\mathrm{Y}$, et al. Anastomotic recurrence of oesophageal squamous cell carcinoma after transthoracic oesophagectomy. Eur J Surg 1998;164(10):759-64.

25. Chu KM M Law SY, Fok M , WongJ. A prospectiverandomized comparison of transhiatal and transthoracic resection for lower- 
third esophageal carcinoma. Am J Surg 1997;174 (3):320-4.

26. Stark SP, Romber MS, Pierce GE, H ermreck AS, Jewell $W R, M$ oran JF, et al. Transhiatal versus transthoracic esophagectomy for adenocarcinoma of the distal esopha- gus and cardia. Am J Surg 1996;172(5):478-81.

27. Pommier RF, Vetto JT, FerrisBL, W ilmarth TJ. Relationships between operativeapproachesand outcomesin esophageal cancer. Am J Surg 1998;175(5):422-5. 